\title{
Kepler-432 b: a massive planet in a highly eccentric orbit transiting a red giant ${ }^{\star}$
}

\author{
S. Ciceri ${ }^{1}$, J. Lillo-Box ${ }^{2}$, J. Southworth ${ }^{3}$, L. Mancini ${ }^{1}$, Th. Henning ${ }^{1}$, and D. Barrado ${ }^{3}$ \\ 1 Max Planck Institute for Astronomy, Königstuhl 17, 69117, Heidelberg, Germany \\ e-mail: ciceri@mpia.de \\ 2 Departamento de Astrofísica, Centro de Astrobiología (CSIC-INTA), 28691 Villanueva de la Cañada, Madrid, Spain \\ 3 Astrophysics Group, Keele University, Staffordshire, ST5 5BG, UK
}

Received 10 October 2014 / Accepted 28 November 2014

\section{ABSTRACT}

\begin{abstract}
We report the first disclosure of the planetary nature of Kepler-432 b (aka Kepler object of interest KOI-1299.01). We accurately constrained its mass and eccentricity by high-precision radial velocity measurements obtained with the CAFE spectrograph at the CAHA 2.2-m telescope. By simultaneously fitting these new data and Kepler photometry, we found that Kepler- $432 \mathrm{~b}$ is a dense transiting exoplanet with a mass of $M_{\mathrm{p}}=4.87 \pm 0.48 M_{\text {Jup }}$ and radius of $R_{\mathrm{p}}=1.120 \pm 0.036 R_{\text {Jup }}$. The planet revolves every $52.5 \mathrm{~d}$ around a $\mathrm{K}$ giant star that ascends the red giant branch, and it moves on a highly eccentric orbit with $e=0.535 \pm 0.030$. By analysing two near-IR high-resolution images, we found that a star is located at 1.1" from Kepler-432, but it is too faint to cause significant effects on the transit depth. Together with Kepler-56 and Kepler-91, Kepler-432 occupies an almost-desert region of parameter space, which is important for constraining the evolutionary processes of planetary systems.
\end{abstract}

Key words. planetary systems - stars: fundamental parameters - stars: individual: Kepler-432

\section{Introduction}

Since its first data release (Borucki et al. 2011a), the Kepler spacecraft has been the most productive planet-hunting mission. It has allowed the discovery of over 4000 exoplanet candidates to date, with a very low false positive frequency at least for small planets (e.g. Marcy et al. 2014; Fabrycky et al. 2014). The falsepositive rate is higher $(\sim 70 \%)$ for Kepler's giant stars (Sliski \& Kipping 2014).

One of the best ways to unequivocally prove the planetary nature of a transiting object is to obtain radial velocity (RV) measurements of the parent star, which also allows precise constraints on the mass of the planet. Unfortunately, the host stars of most of the Kepler candidates are too faint or their RV variation is too small to determine the mass of the planets with current spectroscopic facilities. Nevertheless, considerable effort is made to observationally characterize many interesting Kepler candidates (e.g. Hébrard et al. 2013; Howard et al. 2013; Pepe et al. 2013) and develop new instruments with higher resolution and better performance (see Pepe et al. 2014 for a comprehensive review).

Thanks to the extremely high photometric precision of the Kepler telescope, other methods such as transit-timing variation (e.g. Holman et al. 2010; Steffen et al. 2013; Xie 2013) and orbital brightness modulation (e.g. Charpinet et al. 2012; Quintana et al. 2013; Faigler et al. 2013) have been adopted to confirm the planetary nature of candidate objects. Using the latter method, Huber et al. (2013a) detected two planets in the

* RV data (Table A.1) are only available at the CDS via anonymous ftp to cdsarc.u-strasbg. fr (130.79.128.5) or via http://cdsarc.u-strasbg.fr/viz-bin/qcat?]/A+A/573/L5
Kepler-56 system, while Lillo-Box et al. (2014a) confirmed the hot-Jupiter Kepler-91 b, whose planetary nature was also recently supported by an independent study based on multiepoch high-resolution spectroscopy (Lillo-Box et al. 2014c). Kepler-56 b,c and Kepler-91 b were found to be the first transiting planets orbiting giant stars.

Up to now, more than 50 exoplanets have been detected around evolved giants with Doppler spectroscopy, and their general characteristics are different from those found orbiting main sequence (MS) stars. According to the study of Jones et al. (2014), they are more massive, prefer low-eccentricity orbits, and have orbital semi-major axes of more than $0.5 \mathrm{au}$ with an overabundance of between 0.5 and 0.9 au. Furthermore, the correlation between stellar metallicity and the number of planets seems to be reversed compared with MS stars, even though there is still an open debate on this matter (see discussion in Jones et al. 2014). In this context, the discovery of more exoplanets around evolved stars is vital to enlarge the sample and better characterize the statistical properties of these planetary systems. The cases in which the parent stars are $\mathrm{K}$ or $\mathrm{G}$ giants, which are known to evolve from F- and A-type MS stars, are also very interesting for planet formation and evolution theories and help to form a better demographic picture of planets around early-type stars.

Here we describe the confirmation via RV measurements of the transiting planet Kepler-432 b (aka KOI-1299.01), which we show to be a massive gas giant moving on a very eccentric orbit around an evolved $\mathrm{K}$ giant that is ascending the red giant branch. Both Kepler-432 b and Kepler-91 b are on tight orbits and present physical characteristics that deviate from the systems detected so far by the RV method. 
Table 1. Photometric and physical properties of the host star and transit signal from previous studies.

\begin{tabular}{ccc}
\hline \hline Parameter & Value & Reference \\
\hline$R(\mathrm{mag})$ & 12.135 & NASA Archive \\
$K_{\mathrm{p}}(\mathrm{mag})$ & 12.183 & NASA Archive \\
Depth $(\mathrm{ppm})$ & 914 & NASA Archive \\
Duration $(\mathrm{h})$ & 14.7951 & NASA Archive \\
$R_{\star}\left(R_{\odot}\right)$ & $4.160 \pm 0.120$ & Huber et al. $(2013 \mathrm{~b})$ \\
$M_{\star}\left(M_{\odot}\right)$ & $1.353 \pm 0.101$ & Huber et al. $(2013 \mathrm{~b})$ \\
$\rho_{\star}\left(\mathrm{g} \mathrm{cm}^{-3}\right)$ & $0.02650 \pm 0.00049$ & Huber et al. $(2013 \mathrm{~b})$ \\
\hline
\end{tabular}

Notes. The data taken from the NASA Exoplanet Archive are available on http://exoplanetarchive.ipac.caltech. edu.

\section{Observations and data analysis}

Kepler-432 was continuously monitored by Kepler from May 2009 to May 2013, being observed in all the 17 quarters in longcadence mode and during 8 quarters in short-cadence mode. It was recognized as a Kepler object of interest by Borucki et al. (2011b) after it showed a periodic dimming in the light curve every $52.5 \mathrm{~d}$. A subsequent study of Kepler candidates by Huber et al. (2013b), making use of the asteroseismology technique, refined some parameters of this system. These were updated by Burke et al. (2014). We summarise relevant parameters in Table 1.

\subsection{CAFE data}

In 2012 we started a programme to confirm a subset of Kepler candidates via spectroscopic follow-up observations. For this purpose, we used the Calar Alto Fiber Echelle (CAFE) spectrograph mounted on the $2.2 \mathrm{~m}$ Calar Alto telescope. CAFE is an echelle spectrograph capable of achieving an average resolution of $R=63000 \pm 4000$ in the optical regime. The nominal precision in measuring RVs of stellar objects, tested on known exoplanet host stars (Aceituno et al. 2013; Lillo-Box et al. 2014a), is a few tens of $\mathrm{ms}^{-1}$, sufficient to detect a signal caused by a close-in Jupiter-like planet.

We obtained 28 spectra of Kepler-432 during 16 nights of observations carried out in the 2013 and 2014 seasons, during the best visibility time of the Kepler field. The exposure time was $1800 \mathrm{~s}$ for most spectra, but increased to $2700 \mathrm{~s}$ for six spectra to compensate for the presence of thin clouds and veils. Each spectrum was extracted from the raw data using the pipeline provided by the Calar Alto observatory based on the extant R3D pipeline developed by Sanchez et al. (2006). In brief, each order of a spectrum is extracted from the flat-fielded and debiased science image thanks to a continuum image that traces the orders along the pixels. Each spectrum is calibrated in wavelength using the lines of a ThAr spectrum taken after the science frame.

RV measurements were obtained by cross-correlating each observed spectrum with a synthetic spectrum created from the stellar parameters found in the literature. In particular, the crosscorrelation was made order by order, and the final RV measurement was the median value of all those obtained (Müller et al. 2013). The RV values estimated are listed in Table A.1 together with their relative uncertainties. Finally, to better characterize Kepler-432, we combined several CAFE spectra of the star to obtain one with a high signal-to-noise ratio, from which, following the methodology described in Fossati et al. (2010), we inferred the effective temperature of the star $T_{\text {eff }}$ and its surface
Table 2. Measured properties of sytem Kepler-432.

\begin{tabular}{ll}
\hline \hline Parameter & Value \\
\hline$T_{\text {eff }}(\mathrm{K})$ & $4850 \pm 100$ \\
$\log g(\mathrm{cgs})$ & $3.0 \pm 0.5$ \\
$T_{0}(\mathrm{BJD} / \mathrm{TDB})$ & $2455477.02906 \pm 0.0014$ \\
$P(\mathrm{~d})$ & $52.50097 \pm 0.00021$ \\
$K_{\star}\left(\mathrm{km} \mathrm{s}^{-1}\right)$ & $0.256 \pm 0.021$ \\
$V_{\gamma}\left(\mathrm{km} \mathrm{s}^{-1}\right)$ & $-35.73 \pm 0.014$ \\
$e \cos \omega$ & $0.256 \pm 0.071$ \\
$e \sin \omega$ & $0.469 \pm 0.038$ \\
$e$ & $0.535 \pm 0.030$ \\
$\omega($ degrees $)$ & $61.3 \pm 7.9$ \\
$r_{\star}$ & $0.06374 \pm 0.00039$ \\
$r_{\mathrm{p}}$ & $0.001763 \pm 0.000022$ \\
$i($ degrees $)$ & $88.9 \pm 1.3$ \\
\hline$M_{\mathrm{p}}\left(M_{\text {Jup }}\right)$ & $4.87 \pm 0.48$ \\
$R_{\mathrm{p}}\left(R_{\text {Jup }}\right)$ & $1.120 \pm 0.036$ \\
$g_{\mathrm{p}}\left(\mathrm{m} \mathrm{s}^{-2}\right)$ & $96 \pm 11$ \\
$\rho_{\mathrm{p}}\left(\rho_{\text {Jup }}\right)$ & $3.46 \pm 0.48$ \\
$a($ au $)$ & $0.3034 \pm 0.0089$ \\
\hline
\end{tabular}

gravity $\log g$, see Table 2 . We first fitted the RV data by using the package Systemic Console 2 (Meschiari et al. 2009). To obtain the uncertainties relative to the fitted parameters, we performed bootstrapping and MCMC simulations and adopted the higher values of the uncertainties found with the two methods. The different orbital solutions obtained from the bootstrapping simulations show a clear preference for an eccentric orbit.

As a sanity check, we obtained a Lomb-Scargle periodogram of the RVs without considering the transit times from the photometry. Among the first three peaks we found $P=51.95 \mathrm{~d}$ (with a false-positive probability of 0.0136 ), a value similar to that obtained from the Kepler photometry.

\subsection{Excluding false-positive scenarios}

To rule out the possibility that Kepler-432 is a blended stellar binary system that mimics the observable properties of a transiting planet system, we analysed two high-resolution images of Kepler-432 in $J$ and $K$ bands that were obtained with the NIRC2 imager mounted on the Keck II telescope, used in adaptive optics mode ${ }^{1}$. In these images, there is a clear detection of a nearby star at 1.1 arcsec, which is much fainter than Kepler-432 A, with $\Delta_{J}=5.68 \pm 0.04 \mathrm{mag}$ and $\Delta_{K}=5.19 \pm 0.01 \mathrm{mag}$. We translated these differences into $K_{\mathrm{p}}$-band magnitudes by using the formulae from Howell et al. (2012), obtaining that the component B is $6.68 \pm 0.17 \mathrm{mag}$ fainter than Kepler-432 $\mathrm{A}$ in the Kepler band. Finally, using the relations from Lillo-Box et al. (2014b), we estimated the dilution effect of this faint star on the depth of the transit events, finding a correction of $0.01 R_{\oplus}$ for the radius of the eclipsing object. This correction is much smaller than the uncertainty in our measurement of the radius of Kepler-432 b. Another possibility that we have to consider is that the B component might be an eclipsing binary. However, since we detected an RV signal of a planetary-mass object with the same periodicity as the transit signal and the companion is very faint, this scenario is very unlikely. Instead, the most probable scenario is that the planet is orbiting component $\mathrm{A}$ and that $\mathrm{B}$ only acts as

\footnotetext{
1 The images were published by David Ciardi on the Community Follow-up Observing Program (CFOP), https://cfop.ipac. caltech.edu/home/
} 


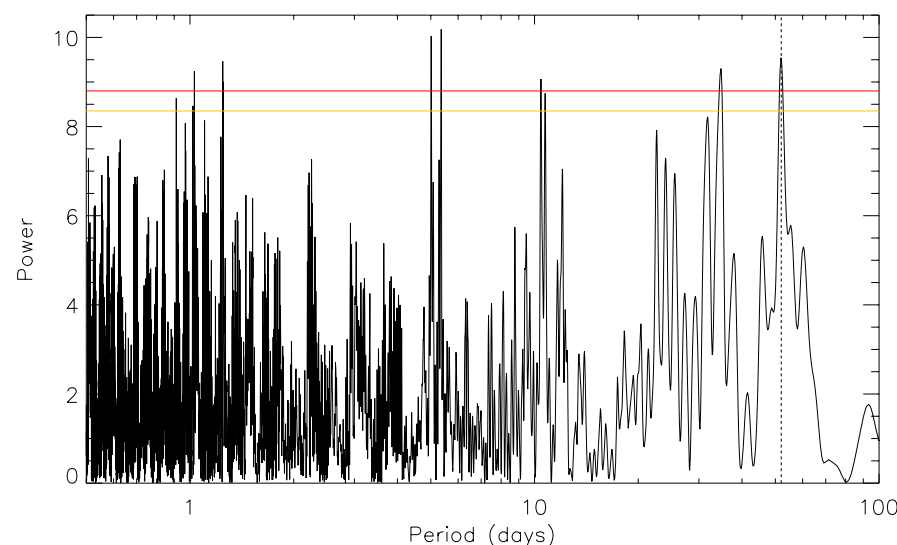

Fig. 1. Lomb-Scargle periodogram obtained from the CAFE RV measurements for Kepler-432. The dashed line highlights the orbital period of Kepler- 432 b. The red and orange lines represent the 5\% and $10 \%$ false-alarm probability.

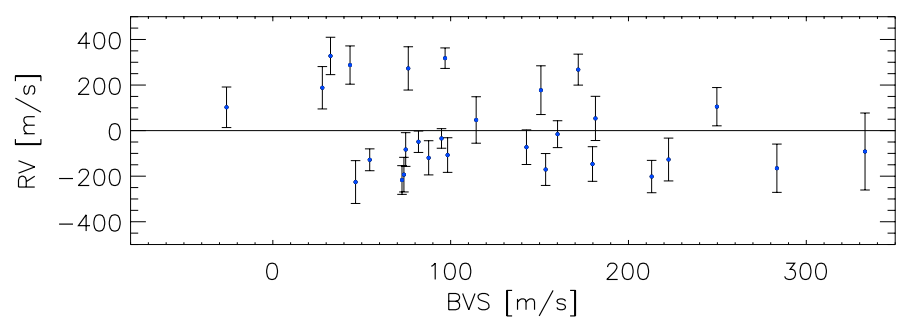

Fig. 2. Radial velocity (RV) versus bisector velocity span (BVS) for Kepler-432.

a diluting source, having very weak implications on the planet (and orbital) properties derived from the light curve.

Another possible source of false positives is stellar activity, which could mimic the presence of a planetary body in the RV signal. To rule out this possibility as well, we determined the bisector velocity span (BVS) from the same spectra from which we obtained the RV measurements. The BVS values are plotted in Fig. 2 together with the best-fitting line, which is consistent with a horizontal line. This means that we did not find any significant correlation between RV and BVS.

\section{Physical properties of the system}

To determine the physical parameters of the system, we simultaneously modelled the Kepler photometry and the CAFE RVs using the JKTEBOP code (see Southworth 2013 and references therein). The parameters of the fit were chosen to be the fractional radii of the two objects $\left(r_{\star}=\frac{R_{\star}}{a}\right.$ and $r_{\mathrm{p}}=\frac{R_{\mathrm{p}}}{a}$ where $a$ is the orbital semi-major axis), orbital inclination $i$, orbital period $P_{\text {orb }}$, reference transit midpoint $T_{0}$, velocity amplitude $K_{\star}$, systemic velocity $V_{\gamma}$ of the star, the eccentricity $(e)$, and argument of periastron $(\omega)$ expressed using the combinations $e \cos \omega$ and $e \sin \omega$.

The Kepler long- and short-cadence data were each converted from flux to magnitude units. Data with more than two transit durations from a transit midpoint (approximately $95 \%$ of the data points for both cadences) were rejected to aid computational efficiency. Each transit was rectified to zero differential magnitude by subtracting a linear or quadratic polynomial trend versus time, fitted to the out-of-transit data points. The shortcadence data were additionally treated by iteratively rejecting $3 \sigma$ outliers, totalling $1.2 \%$ of the data points. Error bars for the data

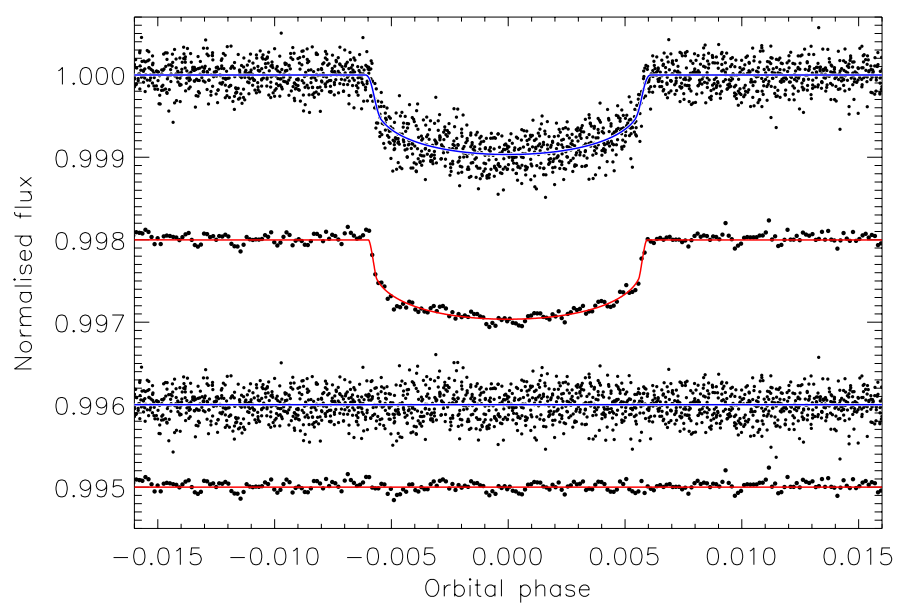

Fig. 3. Kepler long-cadence (top light curve) and short-cadence (bottom light curve) data around transit. The JKTEBOP best fits are shown using solid lines. The residuals of the fits are shown offset towards the base of the figure. We phase-binned the short-cadence data by a factor of 100 to make this plot clearer.

for each cadence were assigned to force a reduced chi-squared of $\chi_{v}^{2}=1.0$. The radial velocity error bars were scaled by $\sqrt{1.8}$ to achieve the same goal.

The very low ratio of the radii means that the transit is shallow and the partial phases (ingress and egress) are short. Their length is poorly determined by the data, leading to $i$ and $r_{\star}$ being highly correlated. The solution is indeterminate without outside constraints. Fortunately, the asteroseismic density from Huber et al. (2013b) can be used to rescue the situation: under the assumption that $M_{\star} \gg M_{\mathrm{p}}$, the density is directly related to $r_{\star}$ (Seager \& Mallén-Ornelas 2003). We therefore fixed $r_{\star}$ at the value for the known density (Table 1) and fitted for $i$. Limb darkening was specified using the quadratic law, whose coefficients were fixed at the theoretical values given by Sing (2010). We also assumed that, neglecting the detected B component, no other light came from the planet or from any additional object along the same sightline. The low sampling rate of the long-cadence data was dealt with as in Southworth (2011).

The best fits are shown in Figs. 3 and 4; the scatters around the best fits were $0.19 \mathrm{mmag}$ and $0.42 \mathrm{mmag}$ for the longand short-cadence. To determine error estimates, we ran Monte Carlo and residual-permutation (Southworth 2008) simulations and adopted the larger of the two error bars for each parameter. We also needed to account for the uncertainty in $r_{\star}$. We did this by calculating solutions with $r_{\star}$ fixed at its asteroseismic value \pm its error bar to determine the effect on each parameter, and added this in quadrature to the uncertainty from the Monte Carlo and residual-permutation simulations.

The result of this process was values for $r_{\star}, r_{\mathrm{p}}, i, P_{\mathrm{orb}}, T_{0}, K_{\star}$, $V_{\gamma}$ and $e$. Independent results were calculated for both cadences and found to be consistent. We adopted those from the shortcadence data because they yield parameter values with a better precision. The final physical properties of the system were then calculated using standard formulae, and the uncertainties were propagated with a Monte Carlo approach. These results are collected in Table 2.

\section{Results and conclusions}

We confirmed the planetary nature of Kepler-432 b, a planet with a mass of $4.87 \pm 0.48 M_{\text {Jup }}$ and a radius of $1.120 \pm 0.036 R_{\text {Jup }}$, 


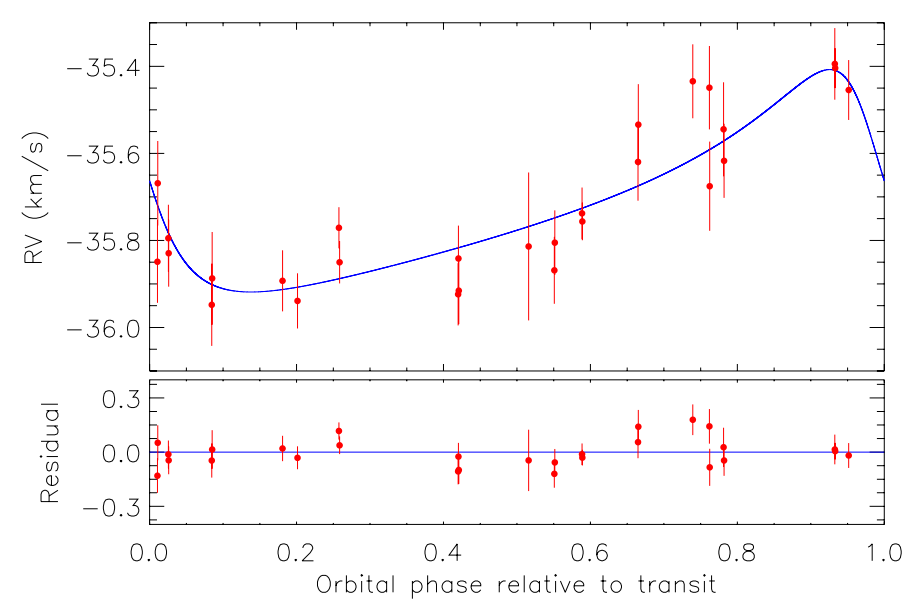

Fig. 4. Upper panel: phased RVs for Kepler-432 red points) and the best fit from JKTEBOP (blue line). Lower panel: residuals of RVs versus best fit.

orbiting a $\mathrm{K}$ giant that is ascending the red giant branch. The planet has an eccentric orbit $(e=0.535 \pm 0.030)$ with a period of $52.50097 \pm 0.00021$ d. After Kepler-56b,c and Kepler-91 b, Kepler-432 b becomes the fourth known transiting planet orbiting an evolved star. These planets have quite different characteristics from those detected by the RV method, and cover regions of parameter spaces that were considered to be deserts until now, see Fig. 5. They are also important indicators of the formation processes and evolutionary scenarios for planets around earlytype stars.

Mazeh et al. (2013) found no significant transit-time variations for Kepler-432, and our RV data do not show any hint of a trend caused by a longer-period companion. More RVs and a longer time-span are necessary to constrain the possible presence of a third body that might be responsible for the location and eccentricity of Kepler-432 b.

Since Kepler-432 A is still evolving and expanding, this planetary system is also very interesting from a dynamical point of view. Currently, the planet reaches the minimum distance of $7.29 \pm 0.52 R_{\star}$ at periastron, while at apastron is $24.08 \pm 0.85 R_{\star}$ away. However, at the end of the red giant branch, the star will have a radius of $\sim 8 R_{\odot}$ and, if we exclude a possible orbital decay due to angular momentum transfer mechanism, the distance of the planet from the star at periastron will be $\sim 3.8 R_{\star}$. This means that the planet will not be devoured by its parent star, but will instead accompany it towards a more distant common fate.

The present Letter was contemporaneously submitted with that by Ortiz et al. (2015), who also confirm the planetary nature of Kepler-432 b.

Acknowledgements. Based on observations obtained with the CAHA $2.2 \mathrm{~m}$ Telescope and the publicly available data obtained with the NASA space satellite Kepler. The Keck images used in this work were taken by David Ciardi. S.C. thanks D. Gandolfi, M. I. Jones, L. Fossati, and M. Ortiz for useful discussion.

\section{References}

Aceituno, J., Sãnchez, S. F., Grupp, F., et al. 2013, A\&A, 552, A31 Borucki, W. J., Koch, D., Basri, G., et al. 2011a, ApJ, 728, 20

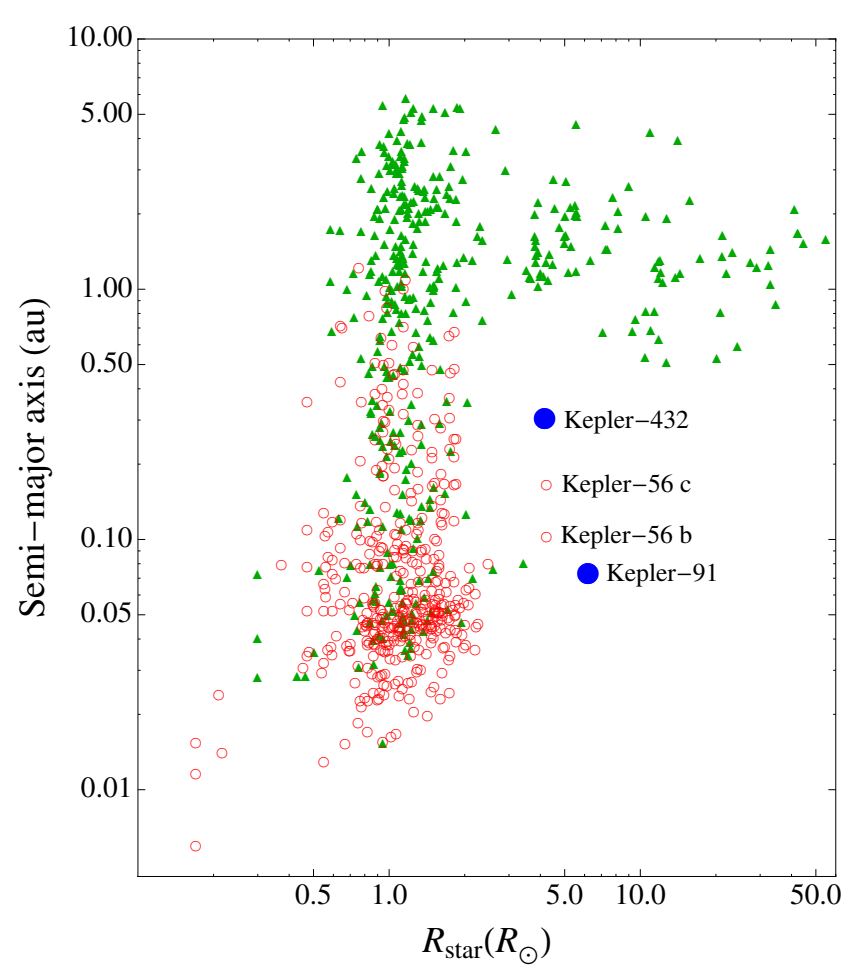

Fig. 5. Stellar radii and semi-major axis of the orbits of known planetary systems. Green triangles denote systems found by the RV method, while red circles are for those found by transit method. Blue points highlight the positions of Kepler-432 and Kepler-91.

Borucki, W. J., Koch, D., Basri, G., et al. 2011b, ApJ, 736, 19

Burke, C. J., Bryson, S. T., Mullally, F., et al. 2014, ApJ, 210, 19

Charpinet, S., Fontaine, G., Brassard, P., et al. 2012, Nature, 480, 496

Fabrycky, D. C., Lissauer, J. J., et al. 2014, ApJ, 790, 146

Faigler, S., Tal-Or, L., Mazeh, T., et al. 2013, ApJ, 771, 26

Fossati, L., Bagnulo, S., Elmasli, A., et al. 2011, ApJ, 720, 872

Hébrard, G., Almenara, J.-M., Santerne, A., et al. 2013, A\&A, 554, A114

Holman, M. J., Fabrycky, D. C., Ragozzine, D., et al. 2010, Science, 330, 51

Howard, A., Sanchis-Ojeda, R., Marcy, G. W., et al. 2013, Nature, 503, 381

Howell, S. B., Rowe, J. F., Bryson, S. T., et al. 2012, ApJ, 746, 123

Huber, D., Carter, J. A., Barbieri, M., et al. 2013a, Science, 342, 331

Huber, D., Chaplin, W. J., Christensen-Dalsgaard, J., et al. 2013b, ApJ, 767, 127

Jones, M. I., Jenkins, J. S., Bluhm, P., et al. 2014, A\&A, 566, A113

Lillo-Box, J., Barrado, D., Moya, A., et al. 2014a, A\&A, 562, A109

Lillo-Box, J., Barrado, D., \& Bouy, H. 2014b, A\&A, 566, A103

Lillo-Box, J., Barrado, D., Henning, Th., et al. 2014c, A\&A, 568, L1

Marcy, G. W., Isaacson, H., Howard, A. W., et al. 2014, ApJS, 210, 20

Mazeh, T., Nachmani, G., Holczer, T., et al. 2013, ApJ, 20816

Meschiari, S., Wolf, A. S., Rivera, E., et al. 2009, PASP, 121, 1016

Müller, A., Roccatagliata, V., Henning, Th., et al. 2013, A\&A, 556, A3

Ortiz, M., Gandolfi, D., \& Reffert, S. 2015, A\&A, 573, L6

Pepe, F., Cameron, A. C., Latham, D. W., et al. 2013, Nature, 503, 377

Pepe, F., Ehrenreich, D., Meyer, M. R. 2014, Nature, 513, 358

Quintana, E. V., Rowe, J. F., Barclay, T., et al. 2013, ApJ, 767, 137

Sánchez, S. F. 2006, Astron. Nachr., 327, 850

Seager, S., \& Mallén-Ornelas, G. 2003, ApJ, 585, 1038

Sing, D. 2010, A\&A, 510, A21

Sliski, D. H., \& Kipping, D. M. 2014, ApJ, 788, 148

Southworth, J. 2008, MNRAS, 386, 1644

Southworth, J. 2011, MNRAS, 417, 2166

Southworth, J. 2013, A\&A, 557, A119

Steffen, J. H., Fabrycky, D. C., Agol, E., et al. 2013, MNRAS, 428, 1077

Xie, J.-W. 2013, ApJS, 208, 22 